\title{
7-Arylbicyclo[4.2.0]oct-1-ene - Synthese durch [2 + 2]-Cycloadditionen von 1,2-Cyclohexadien sowie 1-Methyl-1,2-cyclohexadien und thermische Äquilibrierung der exo/endo-Isomeren
}

\author{
Manfred Christl* und Michael Schreck ${ }^{1)}$ \\ Institut für Organische Chemie der Universität Würzburg, \\ Am Hubland, D-8700 Würzburg
}

Eingegangen am 22. Dezember 1986

\begin{abstract}
Das exo/endo-Isomerenverhältnis $3 \mathbf{a}: \mathbf{3 b}$ bei der bekannten $[2+2]$-Cycloaddition von Styrol an 1,2-Cyclohexadien (2) wurde als temperaturabhängig gefunden. Der Einsatz von (Z)-Deuteriostyrol lieferte den Beweis der Zweistufigkeit dieser Reaktion, und das Diradikal 4 wird als wahrscheinlichste $Z$ wischenstufe angesehen. Erhitzen von $3 \mathrm{~b}$ auf $140-170^{\circ} \mathrm{C}$ führte zur Einstellung des thermodynamischen Gleichgewichts mit 3a $(3 \mathrm{a}: 3 \mathbf{b}=93: 7)$, wobei wieder das Diradikal 4 als Zwischenstufe fungieren dürte. Mit Hilfe kinetischer Messungen ermittelte man die Aktivierungsparameter für das System $3 a \rightleftharpoons 3 b$. - Aus 2 und den Abfangreagenzien p-Methoxystyrol, 1,1-Diphenylethylen sowie 1-Phenylpropen gingen mit bescheidenen Ausbeuten die Titelverbindungen 6a, b, 7 bzw. 8 hervor. Analog zu 2 wurde sein 1-Methylderivat 13 aus 6,6-Dibrom-1-methylbicyclo[3.1.0]hexan (9) durch Methyllithium freigesetzt. In Gegenwart von Styrol entstand neben den Abfangprodukten 14a, b auch das Dimere 12 von 13. - Die 'H-NMR-Spektren der Titelverbindungen belegen eine starre Halbsesselkonformation des Cyclohexenteils mit äquatorial anelliertem Cyclobutanring.
\end{abstract}

7-Arylbicyclo[4.2.0] oct-1-enes - Synthesis by $[2+2]$ Cycloadditions of 1,2-Cyclohexadiene and 1-Methyl-1,2-cyclohexadiene and Thermal Equilibration of the exo/endo Isomers

The exo/endo ratio of the isomers $3 \mathrm{a}, \mathrm{b}$ formed in the known $[2+2]$ cycloaddition of styrene to 1,2-cyclohexadiene (2) has been found to depend on the temperature. The utilization of $(Z)$ deuteriostyrene provided the proof of the stepwise nature of this reaction with the diradical 4 as the most probable intermediate. Heating of $3 \mathrm{~b}$ at $140-170^{\circ} \mathrm{C}$ established the thermodynamic equilibrium between $3 a$ and $3 b(3 a: 3 b=93: \eta)$ and again 4 is assumed to be the intermediate. By means of kinetic measurements the activation parameters of the system $3 a \neq 3 b$ have been determined. - From 2 and the trapping reagents p-methoxystyrene, 1,1-diphenylethylene, and 1-phenylpropene the title compounds $6 \mathrm{a}, \mathrm{b}, 7$, and 8 , respectively, were formed in low yields. In analogy to 2 its 1-methyl derivative 13 has been generated from 6,6-dibromo-1-methylbicyclo[3.1.0]hexane (9) with methyllithium. From the reaction in the presence of styrene the trapping products $14 \mathrm{a}, \mathrm{b}$ were isolated in addition to the dimer 12 of 13 . The 'H NMR spectra of the title compounds support a rigid halfchair conformation of the cyclohexene moiety with the cyclobutane ring anellated equatorially.

her besten Weg zu 2 entdeckt ${ }^{10)}$ und dieses durch Styrol zu 3a, b abgefangen ${ }^{9}$. Für die Reaktionen bei -15 und $40^{\circ} \mathrm{C}$ gaben sie das Verhältnis $3 \mathbf{a}: 3 \mathbf{b}=2.2: 1 \mathrm{an}^{9}$. Wie wir jetzt fanden, hängt dieses Verhältnis jedoch deutlich von der Reaktionstemperatur ab: $4.08: 1$ bei $-45^{\circ} \mathrm{C}, 3.81: 1$ bei $-29^{\circ} \mathrm{C}, 3.18: 1$ bei $-9^{\circ} \mathrm{C}, 2.96: 1$ bei $11^{\circ} \mathrm{C}$ und $2.74: 1$ bei $42^{\circ} \mathrm{C}$.

Als wahrscheinlichster Weg zu 3a, b wurde der zweistufige über das Diradikal 4 vorgeschlagen ${ }^{9}$. Durch Einsatz von $(Z)$-Deuteriostyrol ließ sich kürzlich ein nichtkonzertierter Weg beweisen ${ }^{11}$. Vor der Publikation dieses Ergebnisses haben wir das gleiche Experiment ausgeführt, jedoch benutzten wir anders als Waali et al. ${ }^{11)}$ nicht die ${ }^{2} \mathrm{H}$-, sondern die ${ }^{1} \mathrm{H}$-NMR-Spektroskopie als Analysenmethode. Dabei gaben sich [D]-3a und [D]-3b, die wir gaschromatographisch getrennt haben, jeweils dadurch als 1:1-Isotopomerengemische zu erkennen, daß die Markierung gleichmäßig auf beide 8-Positionen verteilt war (vgl. Diskussion im Abschnitt D). Im Fall einer konzertierten $[2+2]$-Cycloaddition müßte entweder die mit $(Z)$-Deuteriostyrol vorgegebene Stereochemie vollständig erhalten bleiben $\left(\left[\sigma_{\sigma}{ }_{s}+{ }_{\sigma} 2_{s}\right]\right.$-Verlauf) oder vollständig invertiert werden $\left(\left[\sigma^{2}{ }_{s}+\sigma_{2} 2_{a}\right]\right.$-Verlauf), womit [D]-3a und [D]-3b jeweils als sterisch einheitliche Isotopomere anfallen sollten.

Moore und Moser hatten durch Umsetzung von 6,6Dibrombicyclo[3.1.0]hexan (1) mit Methyllithium den bis- 


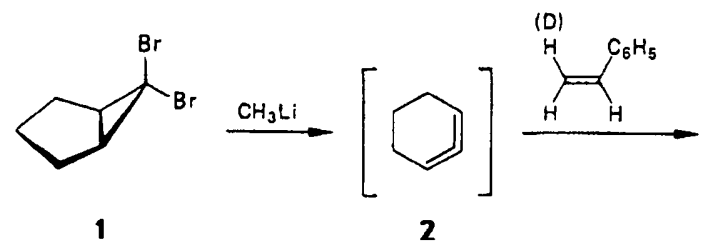

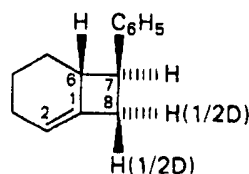

$3 \mathbf{a}([D]-3 \mathbf{a})$<smiles>C1=CCCCC1</smiles>

4

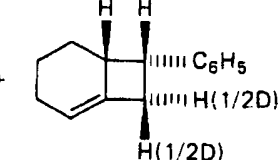

3b $\{[D]-3 b\}$

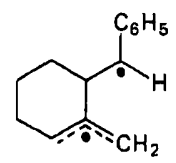

5
Die Abschätzung der Dissoziationsenergie der von C-7 ausgehenden Cyclobutan-Bindungen führt zur Überzeugung, daß sich das Diradikal 4 oder sein Isomeres 5 leicht aus 3 bilden. So gelangt man ausgehend von der Dissoziationsenergie einer vergleichbaren ungespannten CC-Einfachbindung ( $\left.82 \mathrm{kcal} / \mathrm{mol}^{121}\right)$ unter Anrechnung der Benzyl$\left(11.2 \mathrm{kcal} / \mathrm{mol}^{! 3}\right)$ und der Allylradikal-Stabilisierungsenergie $\left(12.8 \mathrm{kcal} / \mathrm{mol}^{13)}\right)$ sowie der Methylencyclobutan-Spannungsenergie $\left(26.9 \mathrm{kcal} / \mathrm{mol}^{14}\right)$ zu einer Energiedifferenz zwischen 3 und 4,5 von ca. $31 \mathrm{kcal} / \mathrm{mol}$.

Tatsächlich wandelte sich ein 20:80-Gemisch aus $3 \mathrm{a}$ und 3b beim Erhitzen auf $140-170^{\circ} \mathrm{C}$ in ein $93: 7-$ Gemisch um, das sich bei weiterem Erhitzen nicht mehr veränderte. Die Messung der Kinetik dieser Gleichgewichtseinstellung und die Auswertung mit der für reversible Reaktionen 1. Ordnung gültigen Gleichung ${ }^{15)}$ lieferte:

$\begin{array}{ccccc}\begin{array}{c}E_{\mathrm{a}} \\ {\left[\mathrm{kcal}^{-1}\right.}\end{array} & \log A & \begin{array}{c}\Delta H^{+} \\ \left.\mathrm{mol}^{-1}\right]\end{array} & \begin{array}{c}\Delta \mathrm{Scal}^{+} \\ \left.\mathrm{mol}^{-1}\right]\end{array} & \begin{array}{c}{[\mathrm{cal} \cdot} \\ \left.\mathrm{mol}^{-1} \cdot \mathrm{K}^{-1}\right]\end{array} \\ \mathbf{3 b} \rightarrow 3 \mathbf{3 a} & 34.5 & 15.8 & 33.7 & 11 \\ \mathbf{3 a} \rightarrow 3 \mathbf{3 b} & 35.4 & 15.1 & 34.6 & 8\end{array}$

Daraus ergeben sich für $\mathbf{3 b} \rightarrow \mathbf{3 a}$ : Reaktionsenthalpie $(\Delta H)=-0.9 \mathrm{kcal} \cdot \mathrm{mol}^{-1}$, Reaktionsentropie $(\Delta S)=3$ $\mathrm{cal} \cdot \mathrm{mol}^{-1} \cdot \mathrm{K}^{-1}$, freie Reaktionsenthalpie $(\Delta G, 432.9 \mathrm{~K})=$ $-2.2 \mathrm{kcal} \cdot \mathrm{mol}^{-1}$.

Wie erwartet, erweist sich das weniger unter Gruppenhäufung leidende exo-Isomere $\mathbf{3 a}$ als thermodynamisch stabiler als das endo-Isomere $\mathbf{3 b}$. Jeweils etwa die Hälfte der Destabilisierung von 3b resultiert bei $432.9 \mathrm{~K}$ aus der ungünstigeren Enthalpie (größere Spannung) und aus der ungünstigeren Entropie (eingeschränktere Rotation der Phenylgruppe).

Die Frage, ob nun Diradikal 4 oder 5 Zwischenstufe bei der Gleichgewichtseinstellung ist, ließ sich nicht direkt beantworten. Für 4 spricht die größere Zahl der Alkylsubstituenten am Allylradikal-Teil, was einen Vorteil von ca. 2 $\mathrm{kcal} / \mathrm{mol}^{131}$ bewirken sollte. Darüber hinaus leidet 5 unter sterischer Kompression, welche die vicinalen Substituenten am Sechsring aufeinander ausüben. Bei 7-Vinylbicyclo[4.2.0]oct-1-enen, den [2 + 2]-Cycloaddukten von 2 an 1,3Butadiene, öffnet sich bei $130-170^{\circ} \mathrm{C}$ im Zuge der Vinylcyclobutan-Cyclohexen-Umlagerung ebenfalls die C-6-C7-Bindung, und Hinweise auf den C-7-C-8-Bindungsbruch wurden nicht gefunden ${ }^{1,16)}$. Da somit dort die zu 5 analoge Diradikal-Zwischenstufe ausscheidet, stützen diese Befunde den Reaktionsweg über das Diradikal 4.

Neben 4 sind auch noch entsprechende Zwitterionen als Intermediate für die Äquilibrierung $\mathbf{3 a} \rightleftharpoons \mathbf{3 b}$ zu diskutieren. Einen Versuch zur Klärung des Sachverhaltes haben wir nicht ausgeführt; jedoch isomerisieren oben erwähnte 7-Vinylbicyclo[4.2.0]oct-1-ene in den Lösungsmitteln Benzol und Acetonitril praktisch gleich schnell ${ }^{13}$, wobei mit hoher Wahrscheinlichkeit die Bildung der Zwischenstufe geschwindigkeitsbestimmend ist. Dies weist auf einen Ubbergangszustand mit ähnlicher Polarität wie die der Edukt-Kohlenwasserstoffe und damit auf eine unpolare, d.h. diradikalische Zwischenstufe. Der Analogieschluß zugunsten von 4 und gegen zwitterionische Alternativen erscheint daher gerechtfertigt.

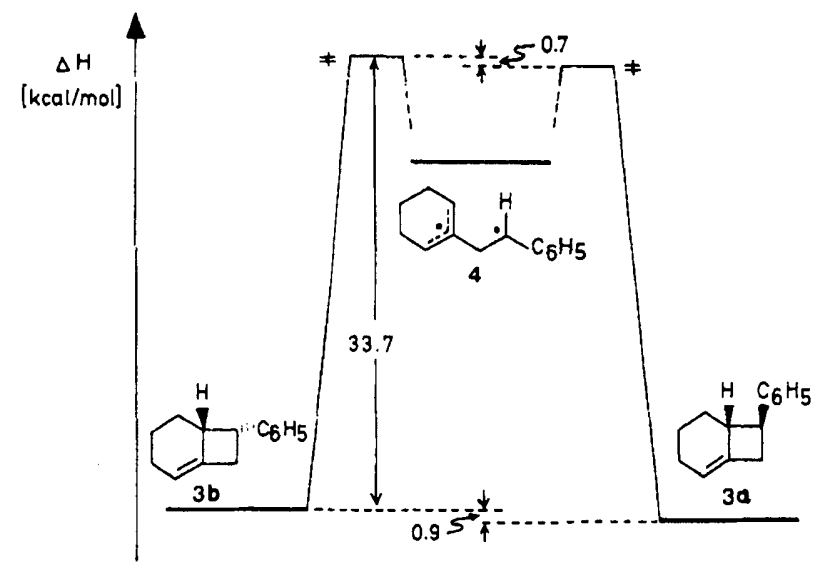

Abb. 1. Enthalpiediagramm für die thermolytische Einstellung des Gleichgewichts zwischen endo- (3b) und exo-7-Phenylbicyclo[4.2.0] oct-1-en (3a)

Durch die kinetischen Messungen zum Gleichgewicht $3 \mathrm{a} \rightleftharpoons \mathbf{4} \rightleftharpoons \mathbf{3} \mathrm{b}$ läßt sich die Enthalpie des höheren der beiden Úbergangszustände $(3 b \rightleftharpoons 4)$ ermitteln. Die niedrigere Barriere $(3 \mathrm{a} \rightleftharpoons 4)$ ist nur durch eine unabhängige Quelle für 4 zugänglich, und diese steht in der Addition von $\mathbf{2}$ an Styrol zur Verfügung. Aus oben schon dargelegter Temperaturabhängigkeit des 3a:3b-Verhältnisses bei der Abfangreaktion von 2 - bei -45 bis $+42^{\circ} \mathrm{C}$ stellt sich das Gleichgewicht zwischen $\mathbf{3 a}$ und $\mathbf{3 b}$ noch nicht ein, so daß die Produktverhältnisse den kinetisch kontrollierten ReaktionsabschluB repräsentieren - errechnet man für $4 \rightarrow 3$ a und $4 \rightarrow 3$ b $\Delta \Delta H^{\ddagger}=0.7 \mathrm{kcal} \cdot \mathrm{mol}^{-1}$ und $\Delta \Delta S^{*}=0 \mathrm{cal} \cdot \mathrm{mol}^{-1}$. $\mathrm{K}^{-1}$. Abb. 1 faßt die Enthalpieverhältnisse zusammen. Die beiden Übergangszustände unterscheiden sich nur in ihrer Enthalpie, wobei die Differenz fast der Enthalpiedifferenz zwischen 3a und $\mathbf{3 b}$ entspricht. Aber im Gegensatz zu den unterschiedlichen Entropien von $\mathbf{3 a}$ und $\mathbf{3 b}$ sind jene der Übergangszustände gleich, was mit dem Hammond-Prinzip im Einklang steht, da es sich bei $3 \mathrm{a} \rightarrow 4$ und $3 \mathrm{~b} \rightarrow 4$ um endotherme Reaktionen handelt. 


\section{B. Addukte von p-Methoxystyrol, 1,1-Diphenyl- ethylen und 1-Phenylpropen an 1,2-Cyclohexadien}

Die Freisetzung von 2 aus 1 in Gegenwart von $p$-Methoxystyrol führte zur Bildung der Bicyclooctene 6a und 6b mit 23\% Ausbeute im Verhältnis 9:1. Im Rahmen der Anwendung der Hammett-Beziehung auf die Addition von Styrolen an 2 war diese Reaktion kürzlich erwähnt worden ${ }^{11}$.

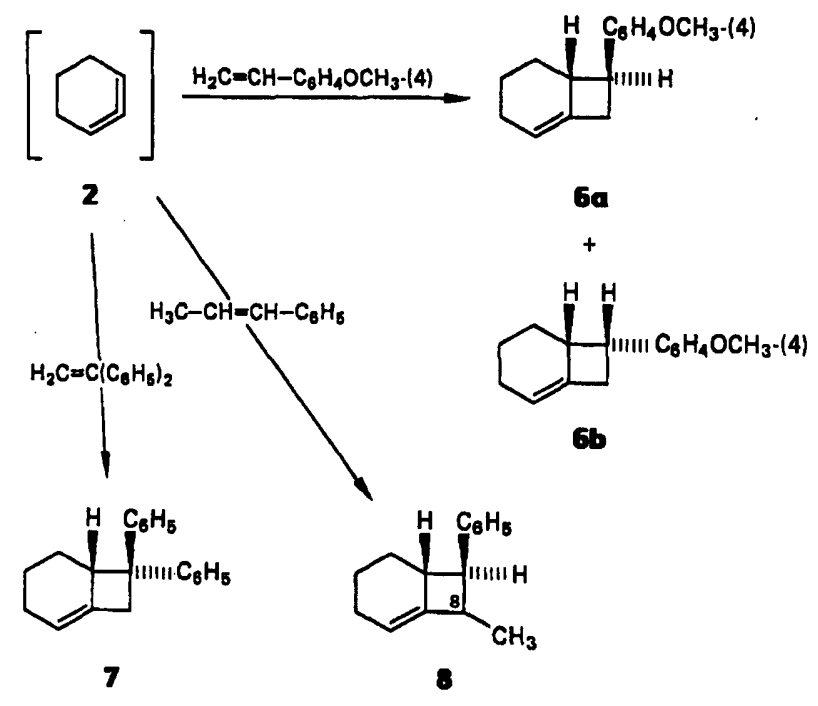

1,1-Diphenylethylen lieferte das einheitliche Addukt $7 \mathrm{mit}$ $12 \%$ Ausbeute. Besonders gering (5\%) war die Ausbeute an 8, dem Anlagerungsprodukt von 1-Phenylpropen. Darin offenbaren sich die Grenzen dieser [2+2]-Cycloaddition, weil trotz der Aktivierung durch die Phenylgruppe ein Substituent an C-2 der Ethylen-Einheit die Reaktionsgeschwindigkeit so verringert, daß 2 in der Hauptsache dimerisiert ${ }^{10}$

\section{Freisetzung von 1-Methyl-1,2-cyclohexadien und Abfangreaktion mit Styrol}

Um den Einfluß eines Substituenten auf die Reaktionsfähigkeit des gespannten Allen-Systems von 2 zu studieren, bezogen wir 1-Methyl-1,2-cyclohexadien (13) in unsere Untersuchungen ein. Es ließ sich der dem Zugang zu 2 analoge Weg über das Addukt 9 von Dibromcarben an 1-Methylcyclopenten beschreiten. Allerdings konnte 9 nur als Gemisch mit seinen Umlagerungsprodukten 10 und 11 im Verhältnis 70:24:6 gewonnen werden (Ausbeute am Gemisch $57 \%$ ).

Die Behandlung von in Styrol gelöstem 9 mit Methyllithium bei $-15^{\circ} \mathrm{C}$ erbrachte mit $92 \%$ Ausbeute ein Gemisch aus dem Dimeren 12 von 13 sowie den [2+2]-Cycloaddukten 14a und 14b im Verhältnis 26:59:15. Die Konfiguration von 12 wurde nicht ermittelt; das analoge Dimere von 2 hat $C_{2}$-Symmetrie ${ }^{10}$. Z Zwar entstand eine erhebliche Menge des Dimeren 12, jedoch ist die Ausbeute an 14a, b mit $54 \%$ immer noch befriedigend. Wahrscheinlich liegt der Dimerisierung der gespannten Allene ein ähnlicher Mechanismus zugrunde wie der Addition an Styrole, so da $\beta$ die Methylgruppe von 13 beide Prozesse in ähnlichem Maße verzögert.
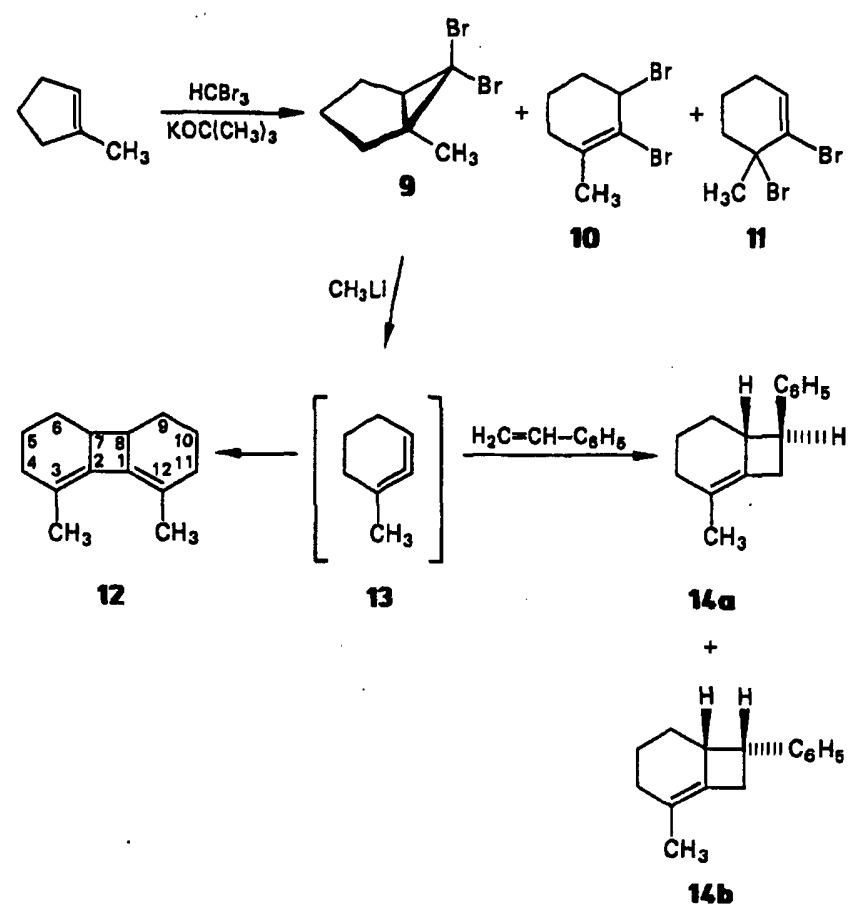

Durch Erhitzen auf $175^{\circ} \mathrm{C}$ wurde die Äquilibrierung von 14a, b erzielt. Das Gleichgewichtsgemisch enthält $92 \% 14$ a und $8 \% 14 \mathrm{~b}$ und ist somit ähnlich aufgeteilt wie bei $3 \mathbf{a}, \mathbf{b}$.

\section{Die NMR-Spektren von 7-Arylbicyclo[4.2.0]oct- 1-enen}

Das Molekülmodell des Bicycloocten-Gerüsts von 3, 6-8 und 14 weist auf eine starre Konformation, in der der Vierring über die äquatoriale Position von C-6 an die Cyclohexen-Halbsesselform anelliert ist (15) und daher 6- $\mathrm{H}$ in die axiale Stellung gezwungen wird. Diese Konformation ist mit den ${ }^{1} \mathrm{H}$-NMR-Spektren bei $400 \mathrm{MHz}$ in Einklang.

Ankerpunkt für die Zuordnung sind zwei Multipletts (ein Dublett von Dubletts von Doppeldubletts und ein Dublett von Doppeltripletts), die bei den endo-7-Arylisomeren 3b, 6b und 14b um $0.45-0.73 \mathrm{ppm}$ bei höherem Feld erscheinen als bei den exo-Verbindungen 3a, 6a bzw. 14a, während andere Signale eine erheblich geringere Variation der chemischen Verschiebungen erfahren. Dieser Hochfeldeffekt ist eine Folge der Abschirmung durch den endo-7-ständigen Arylrest. Laut Konformation 15 kann es sich nur um die Signale der 5- $\mathrm{CH}_{2}-\mathrm{Gruppe}$ handeln, und über die spezifische Zuordnung entscheidet die Größe der Kopplungskonstanten. Bei der Mehrzahl der Signale ist die Feinstruktur so gut aufgelöst, da $B$ alle geminalen und vicinalen Kopplungskonstanten direkt gemessen werden konnten. Sie sind in allen Verbindungen praktisch gleich groß. In Tab. 1 finden sich die gemittelten Werte. Sie entsprechen auf der Basis der Konformation 15 bei Gültigkeit der Karplus-Conroy-Regel der Erwartung. Allylkopplungen zwischen 2-H und 6-H, 8$\mathrm{H}_{\text {endo }}$, 8- $\mathrm{H}_{\text {exo }}$ sowie Homoallylkopplungen zwischen 3- $\mathrm{H}_{\text {endo }}$, 3- $\mathrm{H}_{\text {exo }}$ und 6-H, 8- $\mathrm{H}_{\text {endo }}, 8-\mathrm{H}_{\text {exo }}$ führen zu starken Verbreiterungen der betreffenden Banden und wurden nicht zahlenmäßig erfaßt. 
Tab. 1. Geminale und vicinale Kopplungskonstanten (Absolutwerte in $\mathrm{Hz}$ ) der Protonen am bicyclischen Gerüst von 7 Arylbicyclo[4.2.0]oct-1-enen

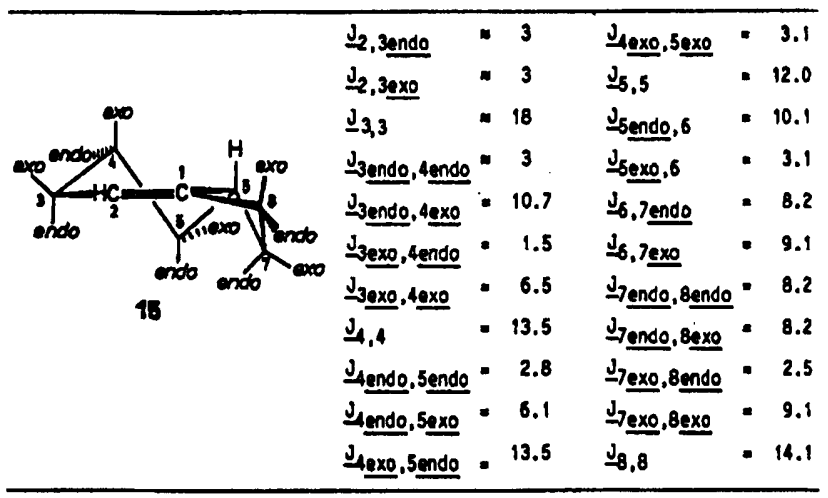

In bezug auf die Deuteriumverteilung in [D]-3b bietet das 7-H-Signal eine sehr zuverlässige Information. Das Spektrum von 3b zeigt ein Triplett $\left(J_{6,70 \times 0}=J_{70 \times 0,8 \times \times 0}=\right.$ $9.1 \mathrm{~Hz})$ von Dubletts $\left(J_{7 e \times 0,8 e n d o}=2.5 \mathrm{~Hz}\right)$. Die gleichmäßige Verteilung der Markierung über beide 8-Positionen in [D]3b wird neben der Intensität der beiden 8-H-Signale, die in etwa der Hälfte der Intensität der 7-H-Bande entspricht, durch die Úberlagerung eines scharfen Tripletts $(9.1 \mathrm{~Hz})$ durch ein breites Dublett $(9.1 \mathrm{~Hz}$, Verbreiterung durch $J_{7 e x o, \text { sendo }}=2.5$ und $J_{7 e x, \mathrm{D}-\mathrm{se} \times 0}=1.4 \mathrm{~Hz}$ ) gleicher Intensität belegt. Beim 1-Phenylpropen-Addukt 8 kann die Konfiguration von C-8 nicht aus den NMR-Spektren abgeleitet werden.

Tab. 2. 'H-NMR-Chemische Verschiebungen ( 8 -Werte) von Bicyclo[4.2.0]oct-1-enen in $\mathrm{CDCl}_{3}$

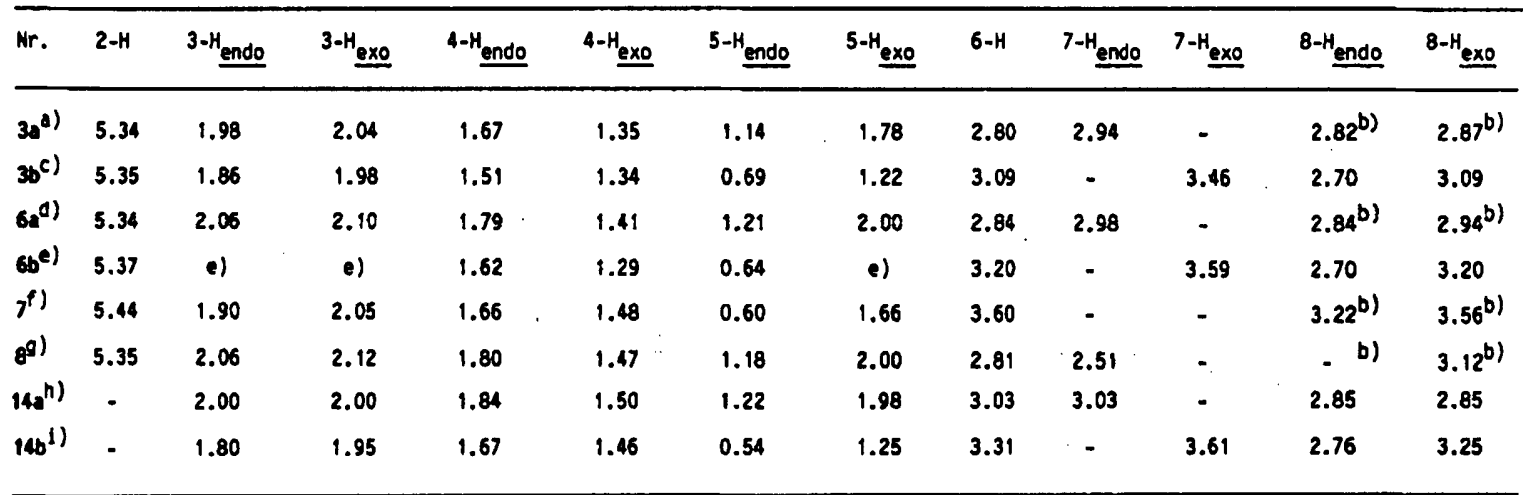

a) $\mathrm{C}_{6} \mathrm{H}_{5}: 7.06(p-\mathrm{H}), 7.10(0-\mathrm{H}), 7.16(m-\mathrm{H})$. - b) Diese Zuordnung ist austauschbar. - $\left.{ }^{\circ}\right) \mathrm{C}_{6} \mathrm{H}_{5}: 7.01-7.21(\mathrm{~m}) .-{ }^{\text {d) }} 4-\mathrm{CH}_{3} \mathrm{OC}_{6} \mathrm{H}_{4}: 3.77(\mathrm{~s})$,

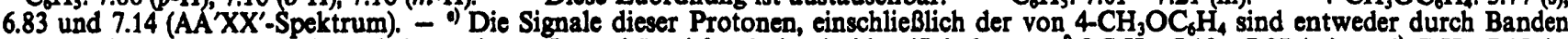
von 6a überlagert oder aufgrund der geringen Intensität nicht eindeutig identifizierbar. $-{ }^{n}{ }_{2} \mathrm{C}_{6} \mathrm{H}_{5}: 7.10-7.37(\mathrm{~m})$. $-8 \mathrm{C}_{6} \mathrm{H}_{5}: 7.19(p-$ H), $7.24(0-\mathrm{H}), 7.29(m-\mathrm{H}) ; \mathrm{CH}_{3}: 1.24(\mathrm{~d}, \mathrm{~J}=6.5 \mathrm{~Hz}) .-{ }^{b} \mathrm{C}_{6} \mathrm{H}_{5}: 7.19(\mathrm{p}-\mathrm{H}), 7.24(0-\mathrm{H}), 7.30(m-\mathrm{H}) ; \mathrm{CH}_{3}: 1.59(\mathrm{~s}) .-{ }^{10} \mathrm{C}_{6} \mathrm{H}_{5}: 7.13(0-\mathrm{H})$, $7.16(p-\mathrm{H}), 7.26(m-\mathrm{H}) ; \mathrm{CH}_{3}: 1.62(\mathrm{~s})$.

Tab. 3. ${ }^{13} \mathrm{C}-\mathrm{NMR}$-Chemische Verschiebungen ( $\delta$-Werte) von Bicyclo[4.2.0]oct-1-enen in $\mathrm{CDCl}_{3}$. Zur Zuordnung der Signale standen offresonance-entkoppelte Spektren zur Verfügung

\begin{tabular}{|c|c|c|c|c|c|c|c|c|c|c|c|c|c|}
\hline Nr. & $c-1$ & $c-2$ & $c-3$ & $C-4$ & C-5 & $C-6$ & C-7 & C-8 & Ipso-c & $\underline{0-C}$ & $m-c$ & $p-c$ & $\mathrm{CH}_{3}$ \\
\hline 3a & 137.80 & 113.44 & 24.88 & 21.31 & 28.13 & 46.07 & 49.93 & 38.87 & 144.47 & 126.27 & 128.06 & 125.76 & - \\
\hline 30 & $\left(140.37^{4)}\right.$ & 114.64 & 24.62 & 20.92 & 22.71 & 41.29 & 46.36 & 36.38 & $\left.141.81^{4}\right\rangle$ & $127.51^{b)}$ & $127.80^{b)}$ & 125.76 & - \\
\hline 68 & $\left(138.16^{4)}\right.$ & 113.55 & 25.07 & 21.50 & 28.30 & 45.73 & 50.38 & 39.35 & $\left(37.01^{9)}\right.$ & 127.47 & 113.80 & 158.05 & 55.25 \\
\hline $6 b$ & c) & 114.77 & 24.81 & 21.11 & 22.92 & 40.72 & 46.58 & 36.89 & c) & 128.64 & c) & c) & 55.15 \\
\hline \multirow[t]{2}{*}{7} & 136.98 & 115.01 & $24.96^{\mathrm{d})}$ & 21.20 & $24.50^{\mathrm{d})}$ & 51.96 & 53.74 & 44.34 & 150.86 & $\left(27.73^{b)}\right.$ & $\left(27.80^{\circ)}\right.$ & $126.94^{(b)}$ & - \\
\hline & & & & & & & & & 143.71 & $\left(27.73^{b)}\right.$ & $\left(28.21^{b)}\right.$ & $125.70^{(b)}$ & - \\
\hline 8 & $144.01^{4)}$ & 111.41 & 24.92 & 22.13 & 28.47 & $47.02^{b)}$ & 54.64 & $47.84^{\mathrm{b})}$ & $144.52^{a)}$ & 126.55 & 128.24 & 125.94 & 17.01 \\
\hline $14 \mathrm{a}$ & 130.34 & 120.78 & 30.28 & 22.94 & 28.62 & 45.97 & 50.00 & 37.33 & 144.95 & 126.47 & 128.16 & 125.79 & 16.96 \\
\hline 146 & 132.88 & 121.63 & 30.15 & 22.48 & 23.27 & 41.70 & 46.62 & 35.18 & 142.23 & $127.71^{81}$ & $\left(28.03^{\mathrm{a})}\right.$ & 125.82 & 16.63 \\
\hline
\end{tabular}

a.b) Diese Zuordnungen sind austauschbar. - o) Diese Signale sind entweder durch Banden von 6a überlagert oder wurden wegen zu geringer Intensität nicht gefunden. 
Die ${ }^{1} \mathrm{H}$ - und ${ }^{13} \mathrm{C}$-NMR-chemischen Verschiebungen der 7-Arylbicyclooctan-Derivate sind in den Tab. 2 bzw. 3 zusammengestellt. Man beachte die unterschiedlichen Wirkungen der endo- und exo-7-Arylgruppen auf die chemischen Verschiebungen von C-1, C-5, C-6, C-7 und C-8. Diagnostisch besonders wertvoll ist der am C-5-Signal beobachtbare $\gamma$-gauche-Effekt einer endo-7-Arylgruppe.

Wir danken dem Fonds der Chemischen Industrie für die Förderung.

\section{Experimenteller Teil}

${ }^{1} \mathrm{H}-\mathrm{NMR}$ und ${ }^{13} \mathrm{C}-\mathrm{NMR}$ : Bruker WM 400. - IR: Beckman AccuLab 4. - MS: Varian MAT CH 7. - Elementaranalysen: Carlo Erba Strumentatione Elemental Analyzer 1106. - Gaschromatographie: Varian Aerograph 1145-1 (analytisch) und 920-01 (präparativ).

Temperaturabhängigkeit des Produktverhältnisses 3a:3b bei der Addition von Styrol an 1,2-Cyclohexadien (2): Die Reaktion von 6,6Dibrombicyclo[3.1.0] hexan (1) mit Methyllithium in Ether in Gegenwart eines großen Styrol-Uberschusses wurde wie beschrieben ausgeführt ${ }^{\text {9) }}$ (Ausb. bei $-15^{\circ} \mathrm{C} 69 \%$, Lit. ${ }^{\text {9l }} 76 \%$ ), jedoch insgesamt bei fünf, mit einem Thermostaten konstant gehaltenen Temperaturen. Nach der Kurzwegdestillation des Produktes bei $50-60^{\circ} \mathrm{C}$ (Bad)/0.001 Torr wurde das Verhältnis 3a:3b gaschromatographisch ermittelt (Bedingungen wie in Lit. ${ }^{9)}, 3 \mathrm{a}$ hat die längere Retentionszeit). Die Ergebnisse sind im allgemeinen Teil beschrieben.

8-Deuterio-exo-7-phenyl- ([D]-3a) und 8-Deuterio-endo-7phenylbicyclo[4.2.0]oct-1-en ([D]-3b): (Z)-Deuteriostyrol, verunreinigt mit $10 \%$ des $(E)$-Isomeren, wurde nach Baldwin und $\mathrm{Kapecki}^{{ }^{17}}$ hergestellt und wie Styrol mit $\mathbf{2}^{9)}$ zur Reaktion gebracht. Das anfallende [D]-3a, b-Gemisch wurde mit Hilfe der präparativen Gaschromatographie (Bedingungen wie bei $\mathbf{3 a}, \mathbf{b}$ in Lit. ${ }^{9}$ ) getrennt. In den $400-\mathrm{MHz}^{-}{ }^{\mathrm{H}} \mathrm{H}$-Spektren zeigten sich im Vergleich $\mathrm{zu}$ jenen von $3 \mathrm{a}, \mathrm{b}$ folgende Veränderungen: $[D]-3 \mathrm{a}: \delta=2.82(\mathrm{~m}$, weitgehend durch das 6-H-Signal bei 2.80 überlagert) und 2.87 (br. $\left.\mathrm{d}, J_{7,8} \approx 8 \mathrm{~Hz}\right)\left(8-\mathrm{H}_{\text {endo }}\right.$ und $8-\mathrm{H}_{\text {exo }}$, Intensität jeweils ca. $\left.0.5 \mathrm{H}\right)$, $2.94\left(\mathrm{t}, J_{6,7}=J_{7,8}=8.2 \mathrm{~Hz} ; 7-\mathrm{H}\right.$ ). [D]-3 b: $\delta=2.70$ (br. s; 8- $\mathrm{H}_{\text {endo }}$, Intensität ca. $0.5 \mathrm{H}$ ), 3.09 (m, durch das 6-H-Signal überlagert; 8$\mathrm{H}_{\text {exo }}$, Intensität ca. $\left.0.5 \mathrm{H}\right), 3.46\left(\mathrm{t}, J_{6,7}=J_{7,8 e x o}=9.1 \mathrm{~Hz} ; 7-\mathrm{H}\right.$ des [endo-8-D]-Isotopomeren, Intensität ca. $0.5 \mathrm{H}$ ) und 3.46 (br. d, $J_{6,7}=9.1 \mathrm{~Hz} ; 7-\mathrm{H}$ des [exo-8-D]-Isotopomeren, Intensität ca. $0.5 \mathrm{H})$.

Kinetische Messungen zur Gleichgewichtseinstellung zwischen 3a und 3b: Ein Gemisch aus 20\% 3a und $80 \%$ 3b wurde in der 10fachen Menge Mesitylen gelöst, i.Vak. entgast, dann mit Stickstoff gesättigt und in ca. zehn mit Stickstoff gefüllte Schmelzpunktröhrchen gebracht. Nach dem Abschmelzen tauchte man diese vollständig in ein thermostatisiertes Olbad (Temperaturschwankungen $\pm 0.1 \mathrm{~K}$ ). In geeigneten Zeitabständen nahm man ein Röhrchen nach dem anderen aus dem Bad, schreckte sie in einem Eisbad ab und analysierte den Inhalt mit Hilfe der Gaschromatographie (Säulentemp. $142^{\circ} \mathrm{C}$, sonst Bedingungen wie in Lit. ${ }^{9}$; die Verweildauer im Injektor und auf der Säule war dabei so kurz, daß das 3a:3bVerhältnis nicht verändert wurde). Die Ausbeute war nahezu quantitativ. Die Auswertung der Meßpunkte erfolgte gemäß der für reversible Reaktionen 1. Ordnung gültigen Gleichung ${ }^{15)}$ anhand eines Rechenprogramms $^{18)}$, das mit der Simplex-Methode ${ }^{19)}$ die Geschwindigkeitskonstanten optimiert. Dabei ergaben sich folgende Geschwindigkeits- $\left(k_{1}\right.$ und $\left.k_{-1}\right)$ und Gleichgewichtskonstanten $(K)$ :

$\begin{array}{cccc}\text { Temp. [K] } & k_{1}\left(\mathrm{~s}^{-1} ; 3 \mathrm{a} \rightarrow 3 \mathrm{~b}\right) & k_{-1}\left(\mathrm{~s}^{-1} ; \mathbf{3 b \rightarrow 3 a}\right) & K \\ 443.2 & 4.56 \times 10^{-3} & 5.81 \times 10^{-2} & 12.7 \\ 432.9 & 1.82 \times 10^{-3} & 2.20 \times 10^{-2} & 12.1 \\ 423.4 & 7.50 \times 10^{-4} & 9.10 \times 10^{-3} & 12.1 \\ 415.4 & 2.90 \times 10^{-4} & 4.29 \times 10^{-3} & 14.5 \\ 413.9 & 2.70 \times 10^{-4} & 3.53 \times 10^{-3} & 13.1\end{array}$

Mit diesen Geschwindigkeitskonstanten errechnete man die im allgemeinen Teil angegebenen Aktivierungsparameter mit Hilfe der Arrhenius- bzw. Eyring-Gleichung. Dabei ergaben sich Korrelationskoeffizienten von $\geqq 0.999$.

exo-7- (6a) und endo-7-(4-Methoxyphenyl) bicyclo[4.2.0]oct-1-en (6b): Unter Stickstoff löste man $12.0 \mathrm{~g}$ (50.0 mmol) 6,6-Dibrombicyclo[3.1.0]hexan (1) in $36.0 \mathrm{~g}$ (268 mmol) 4-Methoxystyrol, kühlte auf $-10^{\circ} \mathrm{C}$ und tropfte unter Rühren innerhalb von 30 min 58.5 mmol Methyllithium ( $50.0 \mathrm{ml} 1.17 \mathrm{M}$ in Ether) zu. Danach ließ man innerhalb von 20 min auf $0^{\circ} \mathrm{C}$ erwärmen, setzte vorsichtig $20 \mathrm{ml}$ Wasser zu, trennte die Phasen, extrahierte die organische Phase mit $20 \mathrm{ml}$ Wasser, trocknete sie über $\mathrm{Na}_{2} \mathrm{SO}_{4}$, verdampfte den Ether i.Vak. und destillierte überschüssiges 4-Methoxystyrol bei $65-67^{\circ} \mathrm{C} / 4.5$ Torr ab. Aus dem Rückstand destillierten bei $60-70^{\circ} \mathrm{C}(\mathrm{Bad}) / 0.001$ Torr $3.92 \mathrm{~g}$ eines farblosen Ols, das hauptsächlich aus 6a, b und dem Dimeren von 2 bestand. Durch Chromatographie (Chromotron, $\mathrm{SiO}_{2}$, Petrolether $30-70^{\circ} \mathrm{C}$ ) wurden $2.47 \mathrm{~g}(23 \%)$ eines 9:1-Gemisches aus $6 \mathrm{a}$ und $6 \mathrm{~b}$ als farblose Flüssigkeit erhalten. - MS $(70 \mathrm{eV}): m / z(\%)=214\left(100, \mathrm{M}^{+}\right), 185(25)$, 172 (31), 171 (60), 134 (26), 121 (93), 91 (42), 77 (26). - 'H-NMR: Tab. 1, 2. $-{ }^{13}$ C-NMR: Tab. 3.

\section{$\mathrm{C}_{15} \mathrm{H}_{18} \mathrm{O}(214.3)$ Ber. C 84.06 H 8.47 Gèf. C 83.94 H 8.84}

7,7-Diphenylbicyclo[4.2.0 Joct-1-en (7): $2.40 \mathrm{~g}$ (10.0 mmol) 1 wurden unter Stickstoff im Gemisch aus $10 \mathrm{ml}$ 1,1-Diphenylethylen und $5 \mathrm{ml}$ Ether gelöst und auf $0^{\circ} \mathrm{C}$ gekühlt. Innerhalb von $30 \mathrm{~min}$ tropfte man unter Rühren $17.5 \mathrm{mmol}$ Methyllithium $(6.7 \mathrm{ml} 2.60 \mathrm{M}$ in Ether) zu und kühlte dabei kontinuierlich so ab, daß 1,1-Diphenylethylen nicht auskristallisierte, aber möglichst schnell $-10^{\circ} \mathrm{C}$ erreicht wurden. Man rührte dann noch $20 \mathrm{~min}$ bei $-10^{\circ} \mathrm{C}$, setzte vorsichtig $15 \mathrm{ml}$ Wasser zu, trennte die Phasen, extrahierte die wäßrige Phase zweimal mit je $5 \mathrm{ml}$ Ether, trocknete die vereinigten organischen Phasen über $\mathrm{CaCl}_{2}$, verdampfte den Ether i.Vak. und destillierte überschüssiges 1,1-Diphenylethylen bei $50^{\circ} \mathrm{C}(\mathrm{Bad}) /$ 0.001 Torr ab. Aus dem Rückstand destillierten bei $125-135^{\circ} \mathrm{C}$ (Bad)/0.001 Torr $930 \mathrm{mg}$ eines farblosen Ols. Durch Chromatographie an Kieselgel mit Dichlormethan als Laufmittel und anschlieBende Destillation (Sdp. wie Rohprodukt) erhielt man $320 \mathrm{mg}$ $(12 \%)$ reines flüssiges $7 .-\mathrm{MS}(70 \mathrm{eV}): m / z(\%)=260\left(100, \mathrm{M}^{+}\right)$, 258 (60), 218 (43), 217 (66), 215 (38), 202 (38), 182 (35), 169 (38), 165 (40), 141 (65), 115 (34), 91 (52). - ${ }^{1}$ H-NMR: Tab. 1, 2. $-{ }^{13}$ C-NMR: Tab. 3.

\section{$\mathrm{C}_{20} \mathrm{H}_{20}(260.4)$ Ber. C 92.26 H 7.74 Gef. C 91.89 H 7.62}

8-Methyl-exo-7-phenylbicyclo[4.2.0]oct-1-en (8): Unter Stickstoff wurden zur gerührten Lösung von $6.00 \mathrm{~g}(25.0 \mathrm{mmol}) 1$ in $5.00 \mathrm{~g}$ (42.3 mmol) 1-Phenylpropen (käufliches Isomerengemisch, Aldrich) innerhalb von $30 \mathrm{~min} 29.9 \mathrm{mmol}$ Methyllithium $(13.0 \mathrm{ml} 2.3 \mathrm{M}$ in Ether) getropft, wobei man die Temperatur ausgehend von $-20^{\circ} \mathrm{C}$ auf $0^{\circ} \mathrm{C}$ ansteigen ließ. Danach rührte man 30 min bei $20^{\circ} \mathrm{C}$, versetzte vorsichtig mit $10 \mathrm{~m}$ l Wasser, trennte die Phasen, extrahierte die wäßrige Phase mit Ether, trocknete die vereinigten organischen Phasen über $\mathrm{CaCl}_{2}$, verdampfte den Ether i. Vak. und destillierte nach Zusatz von etwas Hydrochinon bei $110^{\circ} \mathrm{C}(\mathrm{Bad}) / 22$ Torr überschüssiges 1-Phenylpropen ab. Aus dem Rückstand destillierten bei ca. $100^{\circ} \mathrm{C}(\mathrm{Bad}) / 0.05$ Torr $1.98 \mathrm{~g}$ einer farblosen Flüssigkeit, aus der sich $1.04 \mathrm{~g}(52 \%)$ des Dimeren von 2 kristallin abschieden. Aus 
der Mutterlauge erhielt man mit Hilfe der präparativen Gaschromatographie (Säule wie bei 3a, b9), 160 $\mathrm{C}$ ) $248 \mathrm{mg}(5 \%) 8$ als farblose Flüssigkeit. - MS $(70 \mathrm{eV}): m / z(\%)=198\left(62, \mathrm{M}^{-}\right), 183(80)$, $155(60), 141$ (86), $115(56), 107$ (57), 91 (100), 79 (65). - 'H-NMR: Tab. 1, 2. - ${ }^{13}$ C-NMR: Tab. 3.

$$
\mathrm{C}_{15} \mathrm{H}_{18} \text { (198.3) Ber. C } 90.85 \text { H } 9.15 \text { Gef. C } 90.58 \text { H } 9.48
$$

6,6-Dibrom-1-methylbicyclo[3.1.0] hexan (9): Zu einer Suspension von $7.85 \mathrm{~g}(70.0 \mathrm{mmol}) \mathrm{Kalium}$-tert-butoxid in $100 \mathrm{ml}$ Petrolether $\left(30-70^{\circ} \mathrm{C}\right)$ und $5.15 \mathrm{~g}(62.8 \mathrm{mmol}) 1-$ Methylcyclopenten tropfte man unter Rühren innerhalb von $1.5 \mathrm{~h}$ bei $-10^{\circ} \mathrm{C} 15.60 \mathrm{~g}(61.7$ mmol) Bromoform. Man rührte weitere $15 \mathrm{~min}$ bei $0^{\circ} \mathrm{C}$, setzte $50 \mathrm{ml}$ Wasser zu, trennte die Phasen, extrahierte die organische Phase siebenmal mit je $50 \mathrm{ml}$ Wasser, trocknete sie bei $0^{\circ} \mathrm{C}$ über $\mathrm{Na}_{2} \mathrm{SO}_{4}$ und engte sie bej $0^{\circ} \mathrm{C} / 0.1$ Torr ein. Flüchtige Bestandteile wurden bei $20^{\circ} \mathrm{C} / 0.001$ Torr verdampft und in einer mit flüssigem Stickstoff gekühlten Vorlage kondensiert. Dieses Kondensat $(8.91 \mathrm{~g}$, $57 \%$ ) erwies sich durch sein ${ }^{13} \mathrm{C}-\mathrm{NMR}$-Spektrum, das keine Fremdbande enthielt, als ein reines 70:24:6-Gemisch aus 9 und seinen beiden Umlagerungsprodukten 2,3-Dibrom-1-methylcyclohexen (10) und 2,3-Dibrom-3-methylcyclohexen (11). - ' $\mathrm{H}-\mathrm{NMR}\left(\mathrm{CDCl}_{3}\right.$, $-30^{\circ} \mathrm{C}$, um die weitere Umlagerung von 9 in 10 und 11 zu verhindern): $9: \delta=1.51\left(\mathrm{~s} ; \mathrm{CH}_{3}\right), 1.60-2.35(\mathrm{~m}) .10: \delta=1.83$ (br. s; $\mathrm{CH}_{3}$ ), 4.95 (br. s; 3-H). $11: \delta=2.58$ (br. d, $J_{6,6} \approx 15 \mathrm{~Hz} ; 1 \mathrm{H}$ von $6-\mathrm{H}_{2}$ ), $6.12\left(\mathrm{dd}, J_{1,6}=5.1\right.$ und $\left.3.1 \mathrm{~Hz} ; 1-\mathrm{H}\right)$. Die weiteren Signale von 10 und 11 sind durch die von 9 überlagert. - ${ }^{13} \mathrm{C}-\mathrm{NMR}\left(\mathrm{CDCl}_{3}\right.$, $-30^{\circ} \mathrm{C}$ ): $9: \delta=21.44$ (q, $\left.\mathrm{CH}_{3}\right), 25.57$ (t, C-3), 30.33 (t, C-4), 36.79 (t, C-2), 40.58 (s, C-1), 43.36 (d, C-5), 48.93 (s, C-6). Zum Vergleich die bisher nicht publizierten Werte von $1: \delta=25.82$ (t, C-3), 29.28 (t, C-2), 39.67 (d, C-1), 40.51 (s, C-6). 10: $\delta=17.75$ (t, C-5), 23.98 (q, $\mathrm{CH}_{3}$ ), 32.76 und 33.76 (jeweils $\mathrm{t}, \mathrm{C}-4$ und $\mathrm{C}-6$ ), 57.57 (d, C-3), 119.0 (s, C-2), 138.98 (s, C-1). 11: $\delta=19.16(t, C-5), 27.66(t, C-6)$, 34.61 (q, $\left.\mathrm{CH}_{3}\right), 42.19$ (t, C-4), 67.06 (s, C-3), 129.37 (s, C-2), 132.49 (d, C-1).

2-Methyl-exo-7- (14a) und 2-Methyl-endo-7-phenylbicyclo[4.2.0]oct-1-en (14b) sowie 3,12-Dimethyltricyclo[6.4.0.0.2.7] dodeca2,12-dien (12): Unter Stickstoff tropfte man zur gerührten Lösung von $9.60 \mathrm{~g}$ des Gemisches aus 9,10 und $11(26.4 \mathrm{mmol} \mathrm{9})$ in $35 \mathrm{ml}$ Styrol innerhalb von $1 \mathrm{~h}$ bei $-15^{\circ} \mathrm{C} 40.0 \mathrm{mmol}$ Methyllithium $\left(25.0 \mathrm{ml} 1.6 \mathrm{M}\right.$ in Ether). Unter weiterem Rühren ließ man auf $0^{\circ} \mathrm{C}$ erwärmen, setzte vorsichtig $25 \mathrm{ml}$ gesättigte wäßrige NaCl-Lösung zu, trennte die Phasen, trocknete die organische Phase über $\mathrm{Na}_{2} \mathrm{SO}_{4}$ und engte i. Vak., zuletzt bei $40^{\circ} \mathrm{C} / 0.01$ Torr, ein. Aus dem Rückstand destillierten bei $73-77^{\circ} \mathrm{C} / 0.01$ Torr, $3.79 \mathrm{~g}$ eines farblosen Ols, das laut Gaschromatogramm und ${ }^{13} \mathrm{C}-\mathrm{NMR}$-Spektrum aus $26 \% 12(38 \%$ Ausb.), 59\% 14 a (43\%) und 15\% 14 b (11\%) bestand. Durch präparative Gaschromatographie wurden die reinen Komponenten als farblose Flüssigkeiten isoliert (Säule wie bei $\mathbf{3 a}, \mathbf{b}^{\mathbf{9}}$, 150 C; 12 hat die kürzeste, 14a die längste Retentionszeit).

12: $M S(70 \mathrm{cV}): m / z(\%)=188\left(66, \mathrm{M}^{+}\right), 173(71), 159(34), 145$ (95), $131(100), 117(48), 105$ (42), 91 (72), 79 (32), 77 (33), 41 (33). ${ }^{1} \mathrm{H}-\mathrm{NMR}\left(\mathrm{CDCl}_{3}\right): \delta=1.06\left(6-\mathrm{H}_{\text {endo }}\right), 1.42\left(5-\mathrm{H}_{\text {exo }}\right), 1.72\left(\mathrm{~s}, \mathrm{CH}_{3}\right)$, $1.80\left(5-\mathrm{H}_{\text {endo }}\right), 1.89\left(6-\mathrm{H}_{\text {exo }}\right), 1.93\left(4-\mathrm{H}_{\text {endo }}\right), 2.07\left(4-\mathrm{H}_{\mathrm{c} x o}\right), 2.19(7-\mathrm{H})$; die Kopplungskonstanten und dementsprechend auch die Multiplizitäten sind sehr ähnlich denen der 7-Arylbicyclo[4.1.0]oct-1-ene (siehe Tab. 1, in Formel 15 sind die $\mathrm{C}$-Atome anders numeriert als in 12). $-{ }^{13} \mathrm{C}-\mathrm{NMR}\left(\mathrm{CDCl}_{3}\right): \delta=19.52$ (q, $\left.\mathrm{CH}_{3}\right), 22.84$ (t, C-5), 27.77 und 30.34 (jeweils $t, C \cdot 4$ und C-6), 47.20 (d, C-7), 119.16 (s, C-3), 136.61 (s, C-1).

$\mathrm{C}_{14} \mathrm{H}_{20}$ (188.3) Ber. C 89.29 H 10.71 Gef. C 88.96 H 10.56 14a: MS $(70 \mathrm{eV}): m / z(\%)=198\left(70, \mathrm{M}^{+}\right), 183(46), 155(32), 142$ (48), 141 (56), 129 (30), 115 (30), 107 (50), 94 (31), 91 (100), 79 (72), 77 (34). - 'H-NMR: Tab. 1, 2. - ${ }^{13}$ C-NMR: Tab. 3.

$$
\begin{array}{rrrr}
\mathrm{C}_{15} \mathrm{H}_{18}(198.3) & \text { Ber. C } 90.85 \text { H } 9.15 \\
14 \mathrm{a} & \text { Gef. C } 91.02 \text { H } 9.13 \\
\text { 14b } & \text { Gef. C } 90.55 \text { H } 9.08
\end{array}
$$

14b: MS (70 eV): Praktisch gleich dem von 14a. - 'H-NMR: Tab. 1, 2. - ${ }^{13}$ C-NMR: Tab. 3.

Zur Ermittlung des Verhältnisses $14 \mathrm{a}: 14 \mathrm{~b}$ bei thermodynamischem Gleichgewicht wurden mehrere Proben eines 78:22-Gemisches aus $14 \mathrm{a}$ und $14 \mathrm{~b}$ wie bei den entsprechenden Versuchen mit 3a, b behandelt. Nach $16 \mathrm{~h}$ Erhitzen auf $175^{\circ} \mathrm{C}$ wurde das Verhältnis $14 \mathrm{a}: 14 \mathrm{~b}=92: 8$ bestimmt.

\section{CAS-Registry-Nummern}

1: 2568-36-7 / 2: 14847-23-5 / 2 (Dimeres): 14308-92-0 / 3a: 23115 . 89-1/[D]-3a (Isomer 1): 106988-80-1 / [D]-3a (Isomer 2): 107079 . 31-2 / 3b: 23068-83-9/[D]-3b (Isomer 1): 107079-30-1 / [D]-3b (Isomer 2): 107079-32-3 / 4: 107009-81-4 / 6a: 107009-88-1/ 6b: 106988-81-2 / 7: 106988-82-3 / 8: 106988-83-4 / 9: 106988-84-5 10: $106988-85-6$ / 11: 106988-86-7 / 12: 106988-87-8 / 13: 10698890-3 / 14a: 106988-88-9/14b: 106988-89-0/ $\mathrm{PhCH}=\mathrm{CH}_{2}: 100-$ 42-5 $/ \mathrm{Ph}_{2} \mathrm{C}=\mathrm{CH}_{2}: 530-48.3 /(\mathrm{Z}) \cdot \mathrm{PhCH}=\mathrm{CHD}: 21370-59-2 /(E)-$ $\mathrm{PhCH}=\mathrm{CHD}: 6911-81-5 / \mathrm{PhCH}=\mathrm{CHMe}: 637-50-3 / \mathrm{CHBr}_{3}: 75$. 25-2 / 4- $\mathrm{MeOC}_{6} \mathrm{H}_{4} \mathrm{CH}=\mathrm{CH}_{2}: 637-69-4$ / 1-Methylcyclopentan: $693-89-0$

1) Aus der Diplomarbeit und der Dissertation M. Schreck, Univ. Würzburg, 1983 und 1986.

2) G. Wittig, P. Fritze, Angew. Chem. 78 (1966) 905; Angew. Chem. Int. Ed. Engl. 5 (1966) 846; Liebigs Ann. Chem. 711 (1968) 82.

3) A. T. Bottini, L. L. Hilton, J. Plott, Tetrahedron 31 (1975) 1997.

4) M. Balci, W. M. Jones, J. Am. Chem. Soc. 102 (1980) 7607.

5) sa) C. Wentrup, G. Gross, A. Maquestiau, R. Flammang, Angew. Chem. 95 (1983) 551; Angew. Chem. Int. Ed. Engl. 22 (1983) 542. - 5b) A. Runge, W. Sander, Tetrahedron Lett. 27 (1986) 5835.

6) M. W. Schmidt, R. O. Angus, jr, R. P. Johnson, J. Am. Chem. Soc. 104 (1982) 6838; R. O. Angus, jr., M. W. Schmidt, R. P. Johnson, ibid. 107 (1985) 532.

7) M. Christl, Angew. Chem. 93 (1981) 515; Angew. Chem. Int. Ed. Engl. 20 (1981) 529

${ }^{B}$ M Christl, R. Lang, M. Lechner, Liebigs Ann. Chem. 1980, 980.

9) W. R. Moore, W. R. Moser, J. Org. Chem. 35 (1970) 908.

10) W. R. Moore, W. R. Moser, J. Am. Chem. Soc. 92 (1970) 5469.

11) S. Harnos, S. Tivakornpannarai, E. E. Waali, Tetrahedron Lett. 27 (1986) 3701

12) S. W. Benson, Thermochemical Kinetics, $2^{\text {nd }}$ ed., S. 309, Wiley, New York 1976

13) R. Merényi, Z. Janousek, H. G. Viehe, in Substituent Effects in Radical Chemistry (H. G. Viehe, Z. Janousek, R. Merényi, Eds.), S. 301, D. Reidel Publishing Company, Dordrechi 1986.

14) K. B. Wiberg, Angew. Chem. 98 (1986) 312; Angew. Chem. Int. Ed. Engl. 25 (1986) 312.

15) J. W. Moore, R. G. Pearson, Kinetics and Mechanism, $3^{\text {rd }}$ ed., S. 304, Wiley, New York 1981.

16) M. Christl, M. Schreck, Angew. Chem. 99 (1987), im Druck; Angew. Chem. Int. Ed. Engl. 26 (1987), im Druck.

17) J. E. Baldwin, J. A. Kapecki, J. Am. Chem. Soc. 92 (1970) 4874.

18) Wir danken den Herren Dr. R. Reinhardt und Prof. Dr. H. Quast, Universität Würzburg, für die Úberlassung eines Programms, das noch dem Fall der reversiblen Reaktionen 1. Ordnung angepaßt werden mußte.

19) F. L. Chubb, J. T. Edward, S. C. Wong, J. Org. Chem. 45 (1980) 2315, und zit. Lit.

$[338 / 86]$ 